\title{
Nitrogen-doped carbon/graphene hybrid anode material for sodium-ion batteries with excellent rate capability
}

Huan Liu ${ }^{\text {a }}$, Mengqiu Jia ${ }^{\mathrm{a} * *}$, Bin Cao ${ }^{\mathrm{a}}$, Renjie Chen ${ }^{\mathrm{b}}$, Xinying Lv ${ }^{\mathrm{a}}$, Renjie Tang ${ }^{\mathrm{a}}$, Feng $\mathrm{Wu}^{\mathrm{b}}$ and $\mathrm{Bin} \mathrm{Xu}^{\mathrm{a}, *}$

${ }^{a}$ State Key Laboratory of Organic-Inorganic Composites, Beijing Key Laboratory of Electrochemical Process and Technology for Materials, Beijing University of Chemical Technology, Beijing 100029, China.

b School of Materials Science \& Engineering, Beijing Key Laboratory of Environmental Science and Engineering, Beijing Institute of Technology, Beijing 100081, China

* Corresponding authors. Tel/Fax: 86-10-64434907. E-mail: binxumail@163.com (B. Xu); jiamq@mail.buct.edu.cn (M. Jia)

\begin{abstract}
Nitrogen-doped carbon/graphene (NCG) hybrid materials were prepared by an in-situ polymerization and followed pyrolysis for sodium-ion batteries. The NCG has a large interlayer distance $(0.360 \mathrm{~nm})$ and a high nitrogen content of 7.54 at $\%$, resulting in a high reversible sodium storage capacity of $336 \mathrm{mAh} \mathrm{g}^{-1}$ at $30 \mathrm{~mA} \mathrm{~g}^{-1}$. The NCG shows a sandwich-like structure, i.e. nitrogen-doped carbon nanosheets closely coated on both sides of graphene. The carbon nanosheets shorten the ion
\end{abstract}


diffusion distance, while the sandwiched graphene with high electronic conductivity guarantees fast electron transport, making the NCG exhibit excellent rate capability (94 $\mathrm{mAh} \mathrm{g}^{-1}$ at $5 \mathrm{~A} \mathrm{~g}^{-1}$ ). It also exhibits good cycle stability with a capacity retention of $89 \%$ after 200 cycles at $50 \mathrm{~mA} \mathrm{~g}^{-1}$.

Keywords: Nitrogen-doped carbon; Graphene; Hybrid material; Anode; Sodium ion battery; Electrochemical performance

\section{Introduction}

Sodium-ion batteries (SIBs) have attracted increasing attention for applications in large-scale grid energy storage because of the natural abundance and low cost of sodium resources [1-4]. Current researches in SIBs mainly focus on electrode materials, especially cathode materials $[3,5]$. However, one of the most serious problems in realizing SIBs is the absence of the suitable negative electrodes $[6,7]$. Carbonaceous materials, e.g., graphite and disordered carbon have been commercially utilized as anode materials for Li-ion batteries (LIBs). Unfortunately, the larger ionic radius of $\mathrm{Na}^{+}$ion $\left(1.02 \AA\right.$, $55 \%$ larger than $\mathrm{Li}^{+}$ion) makes it more difficult to be reversibly inserted into and extracted from the host materials. As a result, graphite cannot be utilized as an insertion host of $\mathrm{Na}^{+}$ion due to its small interlayer distance [6]. Disordered carbon appears to be the most suitable anode materials for sodium-ion storage as its large interlayer distance and amorphous structure can facilitate $\mathrm{Na}^{+}$ion insertion-extraction [8-11]. Various precursors, such as sucrose [12], polymer resin [13] and biomass $[14,15]$ have been used to prepare hard carbons for SIBs. The 
specific capacities of the hard carbons are generally in the range of 100-300 $\mathrm{mAh} \mathrm{g}^{-1}$. However, their capacity and high-rate capability still require improvement because of their intrinsically low graphitization [16]. In addition, their cycle stabilities are insufficient for practical application.

Nitrogen-doping has been proved to be an effective strategy for improving the electrochemical performances of carbons for both SIBs and LIBs [17-19]. The doped nitrogen atoms can introduce some active sites for energy storage, resulting in enhanced specific capacities. Moreover, the incorporation of nitrogen atoms into graphitic networks can increase the conductivity of the carbons, which facilitates the rate performances. Fu et al prepared nitrogen doped porous carbon fibers derived from polypyrrole (Ppy) for SIBs, which presented good electrochemical capacity and excellent rate performance [20]. Our group have reported nitrogen-doped mesoporous carbons with a high reversible sodium storage capacity up to $360 \mathrm{mAh} \mathrm{g}^{-1}$ and good rate capability [19].

As a unique 2D carbon materials with large surface area, good chemical stability and excellent electronic conductivity, graphene has been widely used in conducting networks for LIBs and supercapacitors because its high electronic conductivity can guarantee a high rate capability of the electrodes [21-24]. Recently, Yan and co-workers [25] have prepared a hierarchically porous carbon/graphene composite with high sodium storage performance, indicating the advantages of designing a hybrid nanostructure with graphene for carbon anode materials of SIBs.

Herein, we fabricated a sandwich-like nitrogen-doped carbon/graphene (NCG) 
hybrid material by in-situ polymerization reaction and followed pyrolysis of the polyaniline/graphene oxide composites. Benefiting from its unique nanostructure and the doped nitrogen, the obtained NCGs exhibit high reversible capacity, good cycling stability and excellent rate capability, indicating a promising anode material for SIBs.

\section{Experimental}

\subsection{Synthesis and characterization}

Graphene oxide (GO) was prepared though a modified Hummers method. Typically, GO (200 mg) was dispersed in $1 \mathrm{M} \mathrm{HCl}$ solution $(100 \mathrm{~mL})$ with the assistance of ultrasonication for $1 \mathrm{~h}$. Then, $1 \mathrm{M} \mathrm{HCl}(100 \mathrm{~mL})$ with aniline monomer $(2.3 \mathrm{~g})$ was added into the above suspension and sonicated for $30 \mathrm{~min}$. Afterwards, $100 \mathrm{~mL}$ of $0.25 \mathrm{M}$ ammonium persulfate (APS, solvent: $1 \mathrm{M} \mathrm{HCl}$ ) was rapidly added into the above mixture and stirred for $8 \mathrm{~h}$ at $0-5{ }^{\circ} \mathrm{C}$ to obtain PANI-GO composites. Finally, the composite was washed repeatedly and dried in a vacuum oven at $90{ }^{\circ} \mathrm{C}$ or $12 \mathrm{~h}$, then pyrolyzed at $700-900{ }^{\circ} \mathrm{C}$ for $2 \mathrm{~h}$ under flowing nitrogen at a ramping rate of $10{ }^{\circ} \mathrm{C} \min ^{-1}$. The samples prepared at the pyrolysis temperatures of 700,800 , and $900{ }^{\circ} \mathrm{C}$ were denoted as NCG700, NCG800 and NCG900, respectively. For comparison, pure nitrogen-doped carbon derived from polyaniline were prepared by the same procedure at the pyrolysis temperature of $700{ }^{\circ} \mathrm{C}$ (designed as $\mathrm{NC}$ ) without addition of graphene oxide.

The morphology of the samples was observed by scanning electron microscopy (SEM, SUPRA 55) and transmission electron microscopy (TEM, JEOL-2100). The composition and crystalline structure of the samples were characterized by powder 
X-ray diffraction (XRD, Bruker D8 with $\mathrm{Cu}$ Kradiation), and X-ray photoelectron spectroscopy (XPS, ESCALAB 250). Raman spectra was obtained with a Renishaw 1000 Raman spectrometer (514 nm). Nitrogen adsorption/desorption measurement was performed on Micromeritics ASAP 2460 to obtain the porosity parameters of the samples. The specific surface area $\left(\mathrm{S}_{\mathrm{BET}}\right)$ was calculated by the conventional Brunauer-Emmett-Teller method, the total pore volume $\left(\mathrm{V}_{\text {total }}\right)$ was calculated from the adsorbed $\mathrm{N}_{2}$ amount at a relative pressure of 0.99 .

\subsection{Electrochemical measurements}

For electrode preparation, a slurry of $80 \mathrm{wt} \%$ active materials, $10 \mathrm{wt} \%$ carbon black (Super-p), and $10 \mathrm{wt} \%$ PVDF (binder) in NMP was coated onto Cu foil and then dried at $120{ }^{\circ} \mathrm{C}$ for $12 \mathrm{~h}$ in a vacuum oven. Button cells (2025-type) were assembled using the as-prepared electrodes as working electrodes, glass fiber as separator, a $\mathrm{Na}$ foil as counter electrode and $1 \mathrm{M} \mathrm{NaClO}_{4}$ in the mixture of ethylene carbonate (EC) and diethyl carbonate (DEC) (1:1 by volume) as electrolytes in an Ar-filled glovebox (Mikrouna, $\mathrm{H}_{2} \mathrm{O}, \mathrm{O}_{2}<0.1 \mathrm{ppm}$ ). The electrochemical performance of the cells was evaluated by galvanostatic charge-discharge cycle and cyclic voltammetry (CV) between 0.01 and $3.0 \mathrm{~V} v s$. $\mathrm{Na}^{+} / \mathrm{Na}$. The galvanostatic charge-discharge was carried out on a Land BT2000 battery tester and the CV traces were recorded on a CS350 electrochemical workstation.

\section{Results and discussion}

Fig. 1a shows the schematic diagram for the preparation of NCG samples. Firstly, PANI/GO composites were prepared by an in-situ polymerization reaction. The 
aniline monomer was polymerized on the surface of GO nanosheets using ammonium persulfate as the catalyst. Then the PANI/GO composites were thermal treated at high temperatures under nitrogen flowing. During this process, the GO were thermally reduced to graphene nanosheets, while the surface coated PANI were pyrolyzed to carbon with some residual nitrogen atoms attached, resulting in a sandwich-like, nitrogen-doped carbon/graphene hybrid nanocomposite. The nitrogen atoms are expected to attached in the carbon sheets, forming four types of nitrogen configurations (Fig. 1b) [26, 27]: (i) pyridinic-like N (N-6) connecting with two carbon atoms and donating one p electron to the aromatic system, (ii) pyrrolic-like $\mathrm{N}$ (N-5) connecting with two carbon atoms and contributing two electrons to the system, (iii) quaternary-like $\mathrm{N}(\mathrm{N}-\mathrm{Q})$ substituting one carbon atom within the carbon lattice, (iv) nitrogen oxide (N-oxide).
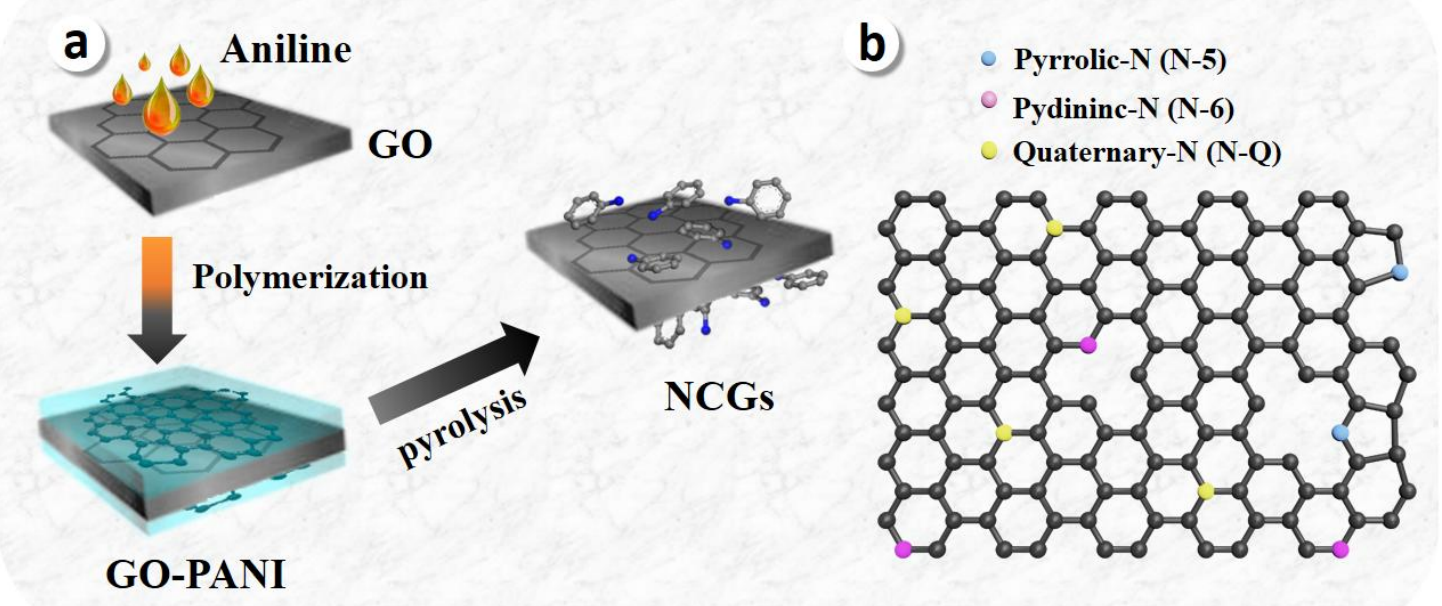

Fig. 1 (a) Schematic diagram for the preparation of NCGs; (b) the bonding configurations of nitrogen functionalities in NCGs.

The morphology of the as-prepared NC and NCGs was characterized by scanning 
electron microscopy (SEM) and transmission electron microscopy (TEM). As shown in Fig. 2a, the nitrogen-doped carbon (NC) derived from pure polyaniline shows agglomeration state of irregular particles. After the addition of GO with nanosheet morphology (Fig. 2b) in aniline polymerization, the PANI/GO-derived NCGs show crumpled lamellar morphology similar to graphene but larger thickness of tens of nanometers and rough surface (Fig. 2c and d). HRTEM image (Fig. 2e) indicates amorphous carbon layers coated on both sides of the graphene nanosheets, revealing the sandwich-like structure of the nitrogen-doped carbon/graphene composites. The sandwiched graphene can enhance the conductivity of the hard carbon, and hence guarantee fast electron transport and good rate capability. Moreover, the interface between graphene and nitrogen-doped carbon can give rise to a large number of defects such as edges (as pointed by the round), which may be active sites for sodium ion storage.

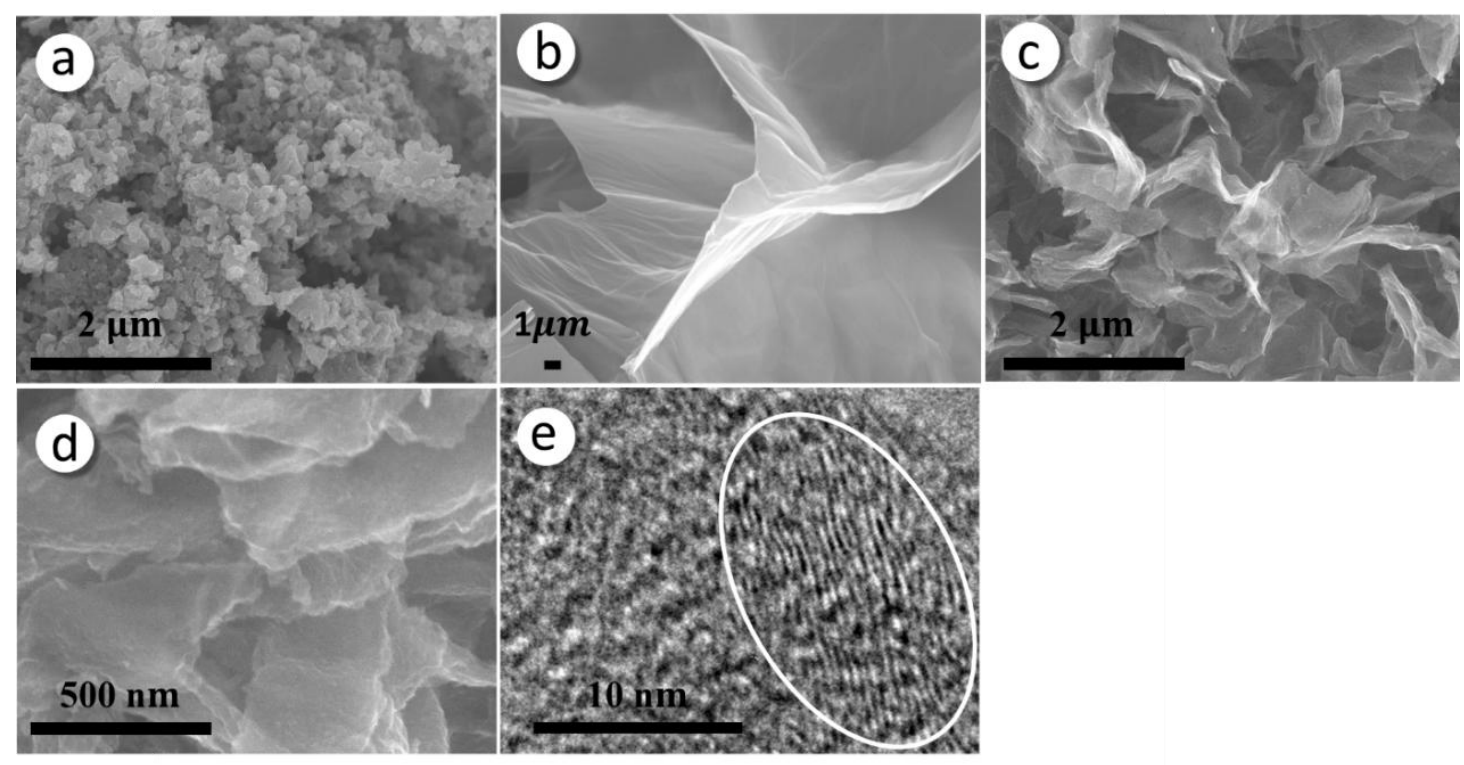

Fig. 2 SEM image of NC (a) and graphene oxide (b); SEM (c, d) and HRTEM (e) images of NCG700. 
Fig. 3a shows the XRD patterns of NC and NCGs prepared at different carbonization temperatures. The XRD patterns of all the samples exhibit two broad peaks indexed to (002) and (101) planes, respectively, indicating typical amorphous carbon. The (002) diffraction in NC locates at $2 \theta=\sim 24.6^{\circ}$, and the interlayer spacing $\left(\mathrm{d}_{002}\right)$ is calculated to be $0.379 \mathrm{~nm}$. For NCG700, the $\mathrm{d}_{002}$ is calculated to be $0.36 \mathrm{~nm}$. With pyrolysis temperature increases, the $\mathrm{d}_{002}$ of NCGs shows a little decrease (Table 1) but all are significantly larger than that of graphite $(0.335 \mathrm{~nm})$, which is essential for the reversible storage of sodium ions. The amorphous crystalline structure of the samples is further confirmed by the two broad peaks of D- and G-bands in the Raman spectra (Fig. 3b). The G peak of the NC appears at $1578 \mathrm{~cm}^{-1}$, and it upshifts to $1590 \mathrm{~cm}^{-1}$ for NCGs, which can be attributed to the restoration of the conjugated structure during pyrolysis process after the addition of graphene [28]. The $\mathrm{I}_{\mathrm{D}} / \mathrm{I}_{\mathrm{G}}$ ratio in the Raman spectra is usually used to evaluate the disordered degree of the carbon materials. The $\mathrm{I}_{\mathrm{D}} / \mathrm{I}_{\mathrm{G}}$ ratio of $\mathrm{NC}$ is 0.92 , which increases to 0.98 for NCG700, implying a more disordered structure. It is supposed some defects and edge planes were introduced by the addition of graphene. $\mathrm{N}_{2}$ sorption $(77 \mathrm{~K})$ were employed to characterize the porous structure of the carbons. As shown in Fig. 3c, all the samples display type IV adsorption/desorption isotherms. The BET surface area of NC is 220 $\mathrm{m}^{2} \mathrm{~g}^{-1}$. The NCGs exhibit lower BET surface areas of $35.1-94.1 \mathrm{~m}^{2} \mathrm{~g}^{-1}$, and shows decreasing tendency with the pyrolysis temperature. XPS are performed to analyze the elemental composition (Fig. 3d), proving the existence of nitrogen in all the NCGs. 

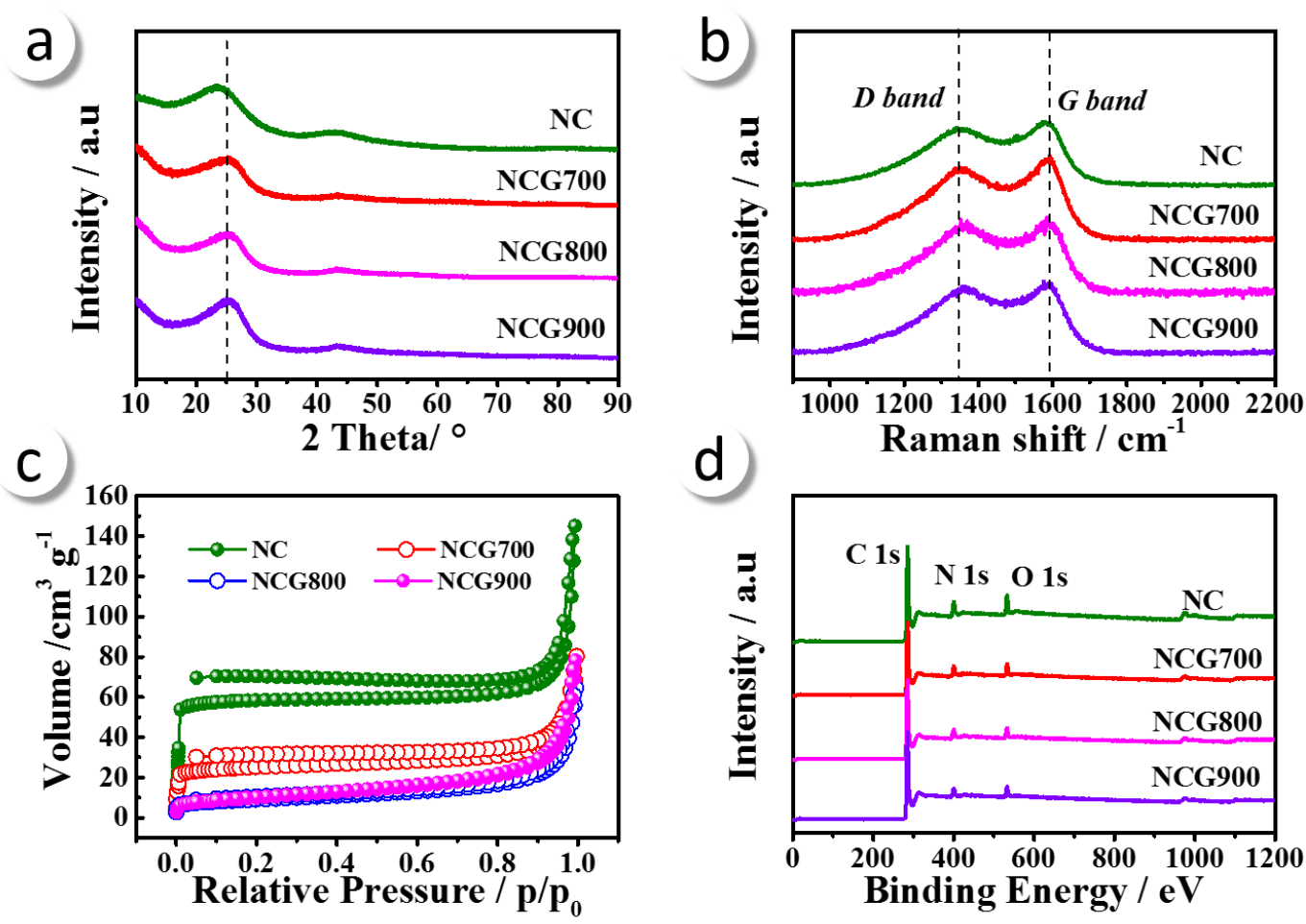

Fig. 3 XRD patterns (a), Raman spectrum (b), nitrogen adsorption/desorption isotherms (c) and XPS spectra (d) of the NC and NCGs prepared at different pyrolysis temperatures.

XPS spectra reveals the NCGs obtained at different pyrolysis temperatures are all rich in nitrogen. As listed in Table 1, NCG700 has similar atomic percentages of nitrogen $(7.54$ at $\%)$ to $\mathrm{NC}(8.32$ at\%). As the pyrolysis temperature increases to $900{ }^{\circ} \mathrm{C}$, the nitrogen content remains 5.68 at\%. As shown in Fig. 4, the nitrogen attached on the carbon can be distinguished into four components by deconvolute the XPS spectra: the pyridinc nitrogen $(\mathrm{N}-6)$ at $398.7 \pm 0.3 \mathrm{eV}$, pyrrolic nitrogen $(\mathrm{N}-5)$ at $400.3 \pm 0.3 \mathrm{eV}$, quaternary nitrogen $(\mathrm{N}-\mathrm{Q})$ at $401.4 \pm 0.5 \mathrm{eV}$, and oxidized nitrogen (N-oxide) between 402 and $405 \mathrm{eV}$ [27]. N-Q atoms will increase the electric conductivity of the graphene layer because it brings extra free electrons, while the N-6 
and N-5 atoms located at the edge of the graphene layer are more effective in adsorbing exotic atoms and enhancing the Na-ion storage capacity [26]. With the pyrolysis temperature increases, the total amount of N-6 + N-5 shows a decreasing tendency but the amount of N-Q increases.
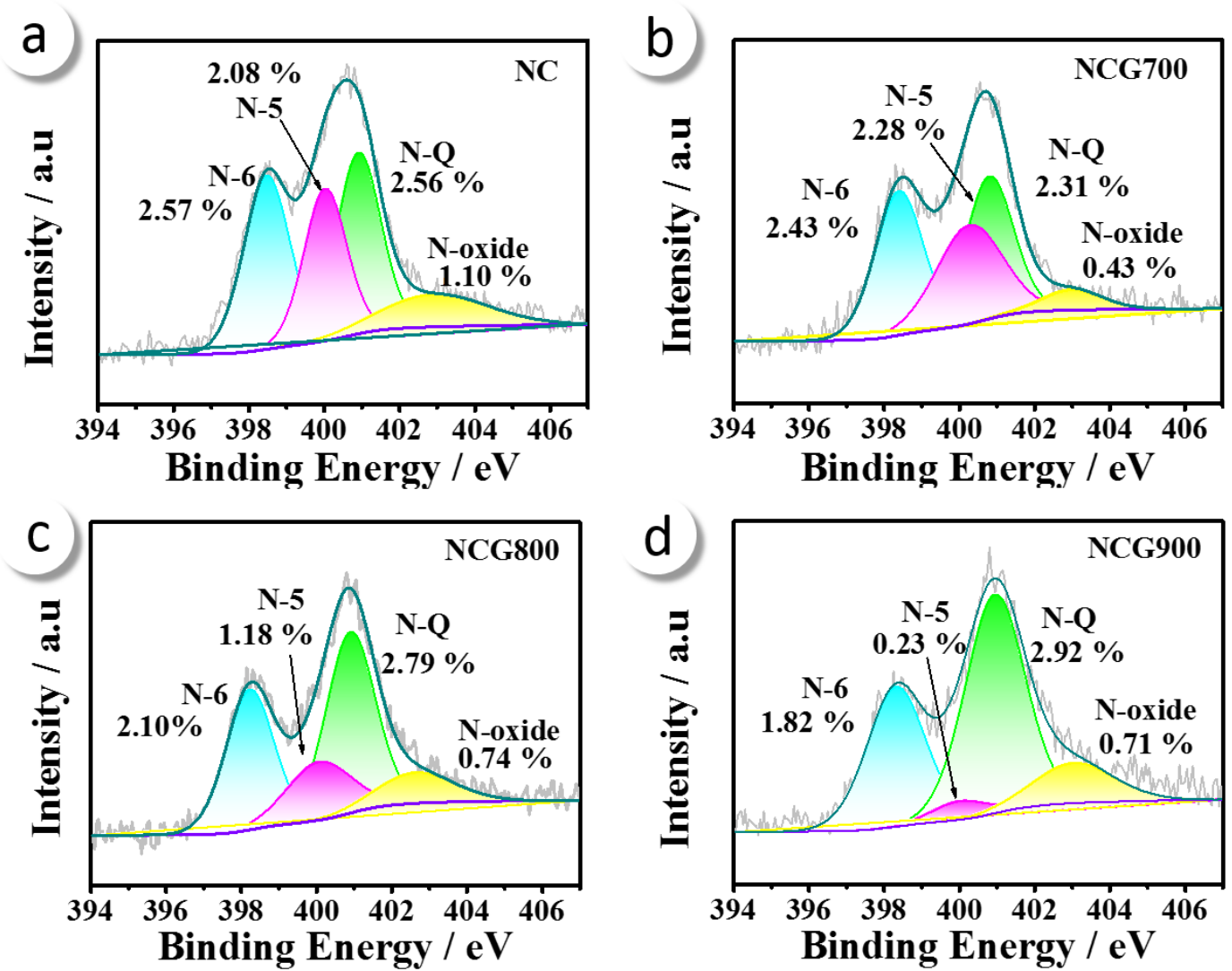

Fig. 4 N 1s spectra of the NC and NCG samples, (a) NC, (b) NCG700, (c) NCG800, (d) NCG900.

The NC and the NCGs prepared at different temperatures show similar charge-discharge profiles between 0.01 and $3.0 \mathrm{~V}$ (Fig. 5), all characteristic of the behavior of the hard carbon anode materials [29, 30]. Thanks to the large interlayer spacing and the numerous defects introduced by nitrogen-doping, all the NC and NCG samples display high reversible capacities above $290 \mathrm{mAh} \mathrm{g}^{-1}$. However, the 
initial coulombic efficiencies of all the sample are around $50 \%$, which is a common phenomenon for hard carbon and is attributed to the severe electrolyte decomposition due to interactions between the electrolyte and the surface species on the carbons [31]. Once a stable solid electrolyte interphase (SEI) layer is constructed during the initial discharge, the coulombic efficiency quickly increases to ca. $100 \%$ in the second and subsequent cycles. NCG700 delivers a reversible capacity of $336 \mathrm{mAh} \mathrm{g}^{-1}$, higher than $297.8 \mathrm{mAh} \mathrm{g}^{-1}$ of NC. The enhanced capacity may be attributed to the more disordered structure of NCG700 as proved by the higher $\mathrm{I}_{\mathrm{D}} / \mathrm{I}_{\mathrm{G}}$ ratio in the Raman spectrum (Fig. 3b). With the pyrolysis temperature increases, the decreased $d_{002}$ and nitrogen content (especially N-6+N-5) make the reversible capacities of NCGs have a little decrease.

a

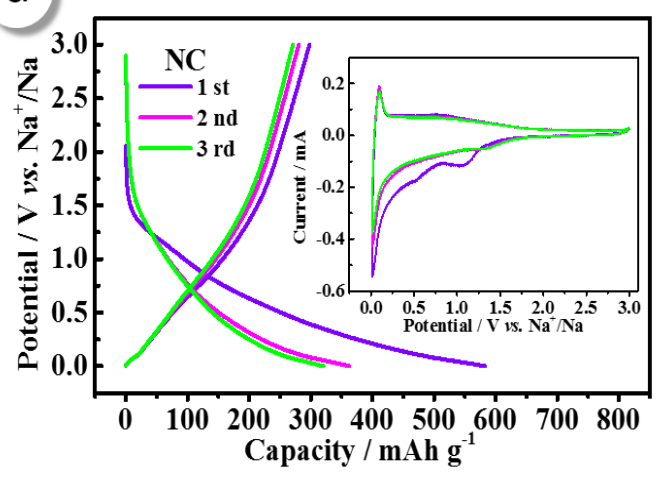

C

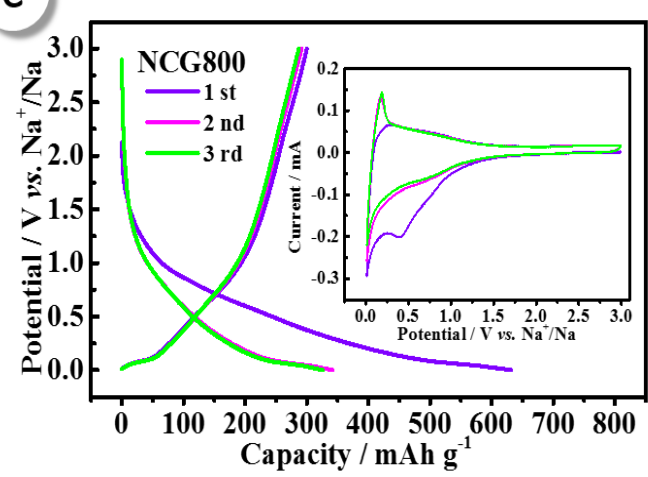

b

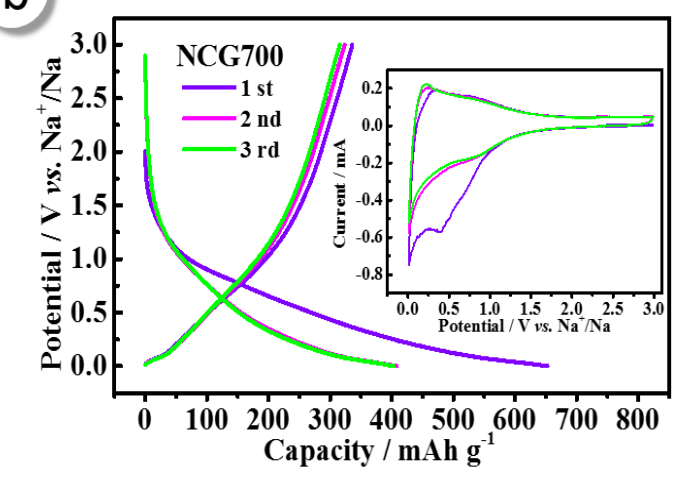

d

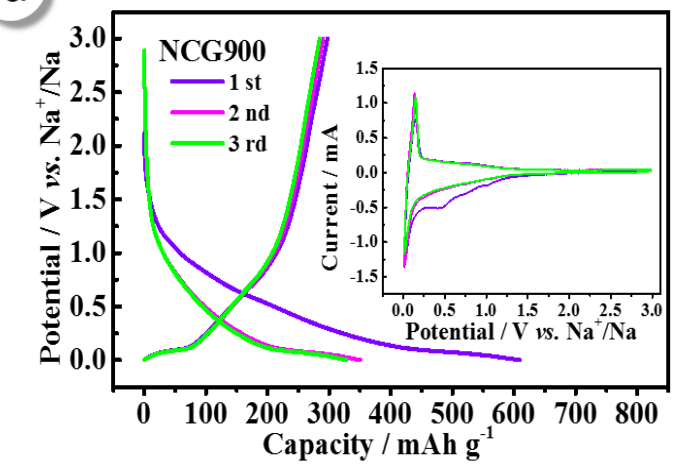

Fig. 5 Charge-discharge and CV (inset; scanning rate of $0.1 \mathrm{mV} \mathrm{s}^{-1}$ ) curves between 
0.01 and $3.0 \mathrm{~V}$ at scan rate of $30 \mathrm{~mA} \mathrm{~g}^{-1}$, (a) NC, (b) NCG700, (c) NCG800, (d) NCG900.

The CV curves of the first three cycles are shown in the inset of Fig. 5a-d. The large irreversible reduction peaks at $0.4 \mathrm{~V}$ in the first cathodic scan can be ascribed to the formation of the solid electrolyte interphase (SEI) film. After the second cycle, the $\mathrm{CV}$ curves almost overlap with each other, indicating good cycle performance of NCGs during sodium ion insertion and extraction. The pair of peaks in the potential range of $0.01-0.2 \mathrm{~V}$ can be assigned to sodium insertion in the interlayer of the graphitic nanocrystallities, and the pair of broad peaks between 0.2 and $1.5 \mathrm{~V}$ correspond to the charge transfer on the surface of small graphitic clusters [32-36]. Additionally, a rectangular shape region can be observed in the potential range of 1.5-3 V, correspond to the capacitive sodium storage behavior, which is helpful to improve the high rate performance. Although NCGs prepared at different pyrolysis temperatures show similar CV curves, some small differences can be observed. With the pyrolysis temperature increases, the peaks in $0.01-0.2 \mathrm{~V}$ become sharp while the wide peaks in $0.2-1.2 \mathrm{~V}$ gradually weaken. This phenomenon may be explained by the more ordered domains with the increase of pyrolysis temperature, agreeing with XRD analysis (Fig. 3a). As the specific surface area decreases with the pyrolysis temperature, the rectangular region characteristic capacitive behavior become weaker and weaker.

Fig. 6a shows the cycle performance of NC and all NCGs at $50 \mathrm{~mA} \mathrm{~g}^{-1}$. The NCG700 exhibits highest reversible capacity and best cycling performance compared 
with NC and other samples. After 200 charge-discharge cycles, NCG700 retains a reversible capacity of $270 \mathrm{mAh} \mathrm{g}^{-1}$, corresponding $89 \%$ of its initial reversible capacity $\left(303 \mathrm{mAh} \mathrm{g}^{-1}\right)$ at $50 \mathrm{~mA} \mathrm{~g}^{-1}$. In contrast, the reversible charge capacity of the $\mathrm{NC}$ fades quickly, from the initial $289 \mathrm{mAh} \mathrm{g}^{-1}$ to only $85 \mathrm{mAh} \mathrm{g}^{-1}$ after 200 cycles, i.e. the capacity retention is only $29 \%$. These results indicate that the addition of graphene sheets is an effective strategy to improve the cycling durability and reversibility of carbon anode materials. Compared the cycling performance of NCGs, it is found that increasing pyrolysis temperatures has a negative effect on the cycling performance and NCG900 shows an obvious capacity fading with prolonged cycling. The better cycling performance of NCG 700 than that of NCG800 and NCG900 may be related to its larger interlayer distance, which facilitates the free insertion/extraction of $\mathrm{Na}^{+}$and can endure volume changes during charge-discharge.
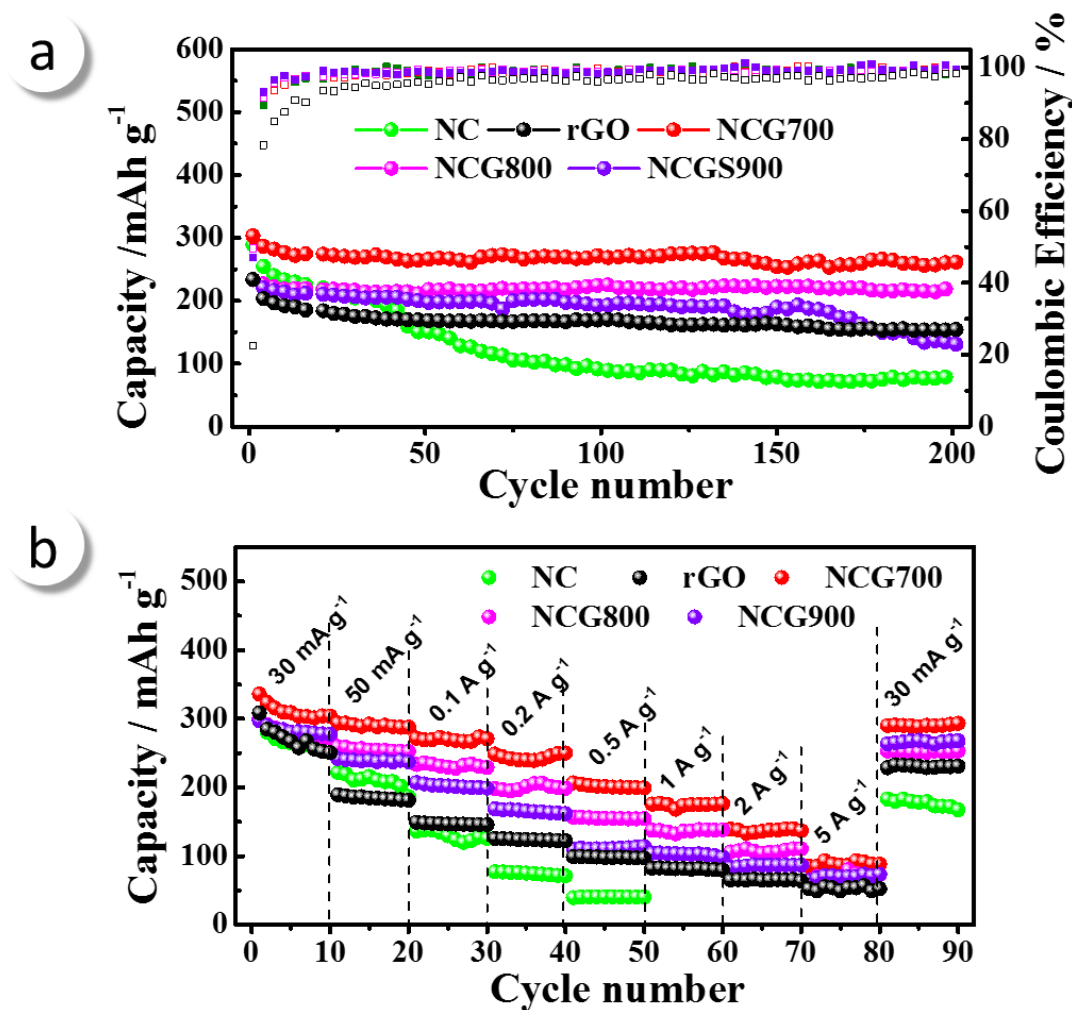
Fig. 6 Cycle performance at a current density of $50 \mathrm{~mA} \mathrm{~g}^{-1}$ (a) and rate performance (b) of NCGs.

As displayed in Fig. 6b, the nitrogen-doped carbon/graphene has superior rate capability to the nitrogen-doped carbon. As the current density increases to $0.5 \mathrm{~A} \mathrm{~g} \mathrm{~g}^{-1}$, the capacity of $\mathrm{NC}$ decays to only $40.8 \mathrm{mAh} \mathrm{g}^{-1}$. However, with graphene nanosheets sandwiched in, the NCG samples exhibit much enhanced rate capacities. For example, the NCG700 can deliver reversible capacities of 336, 274, 249, 207, 177 and 139 $\mathrm{mAh} \mathrm{g}^{-1}$ at the current density of $0.03,0.1,0.2,0.5,1$ and $2 \mathrm{~A} \mathrm{~g}^{-1}$, respectively. Even when the current density increases up to $5 \mathrm{~A} \mathrm{~g}^{-1}$, a capacity as high as $94 \mathrm{mAh} \mathrm{g}^{-1}$ can be remained. When the current density is finally reduced back to $30 \mathrm{~mA} \mathrm{~g}^{-1}$, the average capacity can recover to $294 \mathrm{mAh} \mathrm{g}^{-1}$. The rate performance of NCG700 is very attractive, much superior to some precious reported carboneous anode materials, such as reduced graphene oxide $\left(95.6 \mathrm{mAh} \mathrm{g}^{-1}\right.$ at $\left.1 \mathrm{Ag}^{-1}\right)$ [31], electrospun carbon nanofibers $\left(82 \mathrm{mAh} \mathrm{g}^{-1}\right.$ at $\left.2 \mathrm{~A} \mathrm{~g}^{-1}\right)$ [37] and hollow carbon sphere $\left(75 \mathrm{mAh} \mathrm{g}^{-1}\right.$ at $5 \mathrm{~A}$ $\mathrm{g}^{-1}$ ) [38]. The excellent rate performance of the nitrogen-doped carbon/graphene can be attributed to its unique sandwich-like structure. The carbon nanosheets on both sides of graphene shorten sodium ion diffusion distance, while the sandwiched graphene with high electronic conductivity guarantees fast electron transport.

The nitrogen-doped carbon/graphene hybrid anode material exhibit excellent sodium storage performances, which can be ascribed to the amorphous crystallites and nitrogen doping of the carbon, as well as graphene sandwiched structure. Amorphous 
crystallites with a large interlayer spacing are accessible for $\mathrm{Na}^{+}$insertion, and the defects arisen from both nitrogen heteroatoms and the carbon/graphene interface provide some active sites for $\mathrm{Na}^{+}$storage as well, resulting in high reversible capacity. The sandwiched graphene stabilized the structure of amorphous carbon, and the large interlayer spacing facilitates free insertion/extraction of $\mathrm{Na}^{+}$and can endure volume changes during charge-discharge, which are all beneficial for getting good cycling performances. The carbon nanosheets on both sides of graphene shorten sodium ion diffusion distance, while the sandwiched graphene with high electronic conductivity guarantees fast electron transport, making the NCGs have excellent rate capability.

\section{Conclusion}

Nitrogen-doped carbon/graphene hybrid materials prepared by an in-situ polymerization and followed pyrolysis were used as anode materials for sodium-ion batteries. The NCG has large interlayer spacing, high nitrogen content, and a sandwich-like morphology, i.e. nitrogen-doped carbon sheets coated on both sides of conductive graphene. The unique composition and nanostructure of the NCG enable it exhibit high reversible sodium storage capacity, good cycle durability and excellent rate capability. Therefore, a hybrid nanostructure of nitrogen-doped carbon and graphene is one of the most promising candidates as anode materials for high-performance sodium ion batteries.

\section{Acknowledgement}

This work was financially supported by the National Key Basic Research and 
Development Program (2015CB251100), the National Science Foundation of China (51572011, 21073233, 50802112) and the Fundamental Research Funds for the Central Universities (buctrc201410).

\section{References}

[1] N. Yabuuchi, K. Kubota, M. Dahbi, S. Komaba, Research development on sodium-ion batteries, Chem. Rev. 114 (2014) 11636-11682.

[2] H. Pan, Y.-S. Hu, L. Chen, Room-temperature stationary sodium-ion batteries for large-scale electric energy storage, 6 (2013) 2338-2360.

[3] M. D. Slater, D. Kim, E. Lee, C. S. Johnson, Sodium-ion batteries, Adv. Funct. Mater. 23 (2013) 947-958.

[4] S. Yuan, X. L. Huang, D. L. Ma, H. G. Wang, F. Z. Meng, X. B. Zhang, Engraving copper foil to give large-scale binder-free porous $\mathrm{CuO}$ arrays for a high-performance sodium-ion battery anode, Adv. Mater. 26 (2014) 2273-2279.

[5] S. Yuan, Y. B. Liu, D. Xu, D. L. Ma, S. Wang, X. H. Yang, Z. Y. Cao, X. B. Zhang, Pure single-crystalline $\mathrm{Na}_{1.1} \mathrm{~V}_{3} \mathrm{O}_{7.9}$ nanobelts as superior cathode materials for rechargeable sodium-ion batteries, Adv. Sci. 2 (2015) 1400018.

[6] M. Dahbi, N. Yabuuchi, K. Kubota, K. Tokiwa, S. Komaba, Negative electrodes for Na-ion batteries, Phys. Chem. Chem. Phys. 16 (2014) 15007-15028.

[7] H. Kang, Y. Liu, K. Cao, Y. Zhao, L. Jiao, Y. Wang, H. Yuan, Update on anode materials for Na-ion batteries, J. Mater. Chem. A 3 (2015) 17899-17913.

[8] D. A. Stevens, J. R. Dahn, The mechanisms of lithium and sodium insertion in 
carbon materials, J. Electrochem. Soc. 148 (2001) A803-A811.

[9] Y. Li, S. Xu, X. Wu, J. Yu, Y. Wang, Y.-S. Hu, H. Li, Amorphous monodispersed hard carbon micro-spherules derived from biomass as a high performance negative electrode material for sodium-ion batteries, J. Mater. Chem. A 3 (2015) 71-77.

[10] X. Zhou, Y. Guo, Highly disordered carbon as a superior anode material for room-temperature sodium-ion batteries, ChemElectroChem. 1 (2014) 83-86.

[11] W. Li, M. Zhou, H. Li, K. Wang, S. Cheng, K. Jiang, A high performance sulfur-doped disordered carbon anode for sodium ion batteries, Energy Environ. Sci. 8 (2015) 2916-2921.

[12] T. Chen, L. Pan, T. Lu, C. Fu, D. H. C. Chua, Z. Sun, Fast synthesis of carbon microspheres via a microwave-assisted reaction for sodium ion batteries, J. Mater. Chem. A 2 (2014) 1263-1267.

[13] J. Liu, H. Liu, T. Yang, G. Wang, M. O. Tade, Mesoporous carbon with large pores as anode for Na-ion batteries, Chinese Sci. Bull. 59 (2014) 2186-2190.

[14] W. Lv, F. Wen, J. Xiang, J. Zhao, L. Li, L. Wang, Z. Liu, Y. Tian, Peanut shell derived hard carbon as ultralong cycling anodes for lithium and sodium batteries, Electrochim. Acta 176 (2015) 533-541.

[15] J. Jin, B. Yu, Z. Shi, Lignin-based electrospun carbon nanofibrous webs as free-standing and binder-free electrodes for sodium ion batteries, J. Power Sources 272 (2014) 800-807.

[16] Y. Kim, K. H. Ha, S. M. Oh, K. T. Lee, High-capacity anode materials for sodium-ion batteries, Chem.-Eur. J. 20 (2014) 11980-11992. 
[17] Y. Mao, H. Duan, B. Xu, L. Zhang, Y. Hu, C. Zhao, Z. Wang, L. Chen, Y. Yang, Lithium storage in nitrogen-rich mesoporous carbon materials, Energy Environ. Sci. 5 (2012) 7950-7955.

[18] H. Liu, M. Jia, N. Sun, B. Cao, R. Chen, Q. Zhu, F. Wu, N. Qiao, B. Xu, Nitrogen-rich mesoporous carbon as anode material for high-performance sodium-ion batteries, ACS Appl. Mater. Inter. 7 (2015) 27124-27130.

[19] Z. Guan, H. Liu, B. Xu, Gelatin-pyrolyzed mesoporous carbon as a high-performance sodium-storage material, J. Mater. Chem. A 3 (2015) 7849-7854.

[20] L. Fu, K. Tang, K. Song, P. A. van Aken, Y. Yu, J. Maier, Nitrogen doped porous carbon fibres as anode materials for sodium ion batteries with excellent rate performance, Nanoscale 6 (2014) 1384-1389.

[21] M. Zhang, Y. Wang, M. Jia, Three-dimensional reduced graphene oxides hydrogel anchored with ultrafine $\mathrm{CoO}$ nanoparticles as anode for lithium ion batteries, Electrochim. Acta 129 (2014) 425-432.

[22] M. Kim, Y. wang, J. Kim, Graphene $/ \mathrm{MnO}_{2}$-based composites reduced via different chemical agents for supercapacitors, J. Power Sources 239 (2013) 225-233.

[23] Z. L. Wang, D. Xu, H. G. Wang, Z. Wu, X. B. Zhang, In situ fabrication of porous graphene electrodes for high-performance energy storage, ACS Nano 7 (2013) 2422-2430.

[24] X. L. Huang, R. Z. Wang, D. Xu, Z. L. Wang, H. G. Wang, J. J. Xu, Z. Wu, Q. C. Liu, Y. Zhang, X. B. Zhang, Homogeneous $\mathrm{CoO}$ on graphene for binder-free and ultralong-life lithium ion batteries, Adv. Funct. Mater. 23 (2013) 4345-4353. 
[25] Y. Yan, Y. X. Yin, Y. G. Guo, A sandwich-like hierarchically porous carbon/graphene composite as a high-performance anode material for sodium-ion batteries, Adv. Energy Mater. 4 (2014) 1301584.

[26] W. Shen, C. Wang, Q. Xu, H. Liu, Y. Wang, Nitrogen-doping-induced defects of a carbon coating layer facilitate Na-storage in electrode materials, Adv. Energy Mater. 5 (2015) 1400982.

[27] Z. H. Sheng, L. Shao, J. J. Chen, W. J. Bao, F. B. Wang, X. H. Xia, Catalyst-free synthesis of nitrogen-doped graphene via thermal annealing graphite oxide with melamine and its excellent electrocatalysis, ACS Nano 5 (2011) 4350-4358.

[28] D. Wei, Y. Liu, Y. Wang, H. Zhang, L. Huang, G. Yu, Synthesis of N-doped graphene by chemical vapor deposition and its electrical properties, Nano Lett. 9 (2009) 1752-1758.

[29] Z. Wang, L. Qie, L. Yuan, W. Zhang, X. Hu, Y. Huang, Functionalized N-doped interconnected carbon nanofibers as an anode material for sodium-ion storage with excellent performance, Carbon 55 (2013) 328-334.

[30] J. Zhu, C. Chen, Y. Lu, Y. Ge, H. Jiang, K. Fu, X. Zhang, Nitrogen-doped carbon nanofibers derived from polyacrylonitrile for use as anode material in sodium-ion batteries, Carbon 94 (2015) 189-195.

[31] Y. X. Wang, S. L. Chou, H. K. Liu, S. X. Dou, Reduced graphene oxide with superior cycling stability and rate capability for sodium storage, Carbon 57 (2013) 202-208.

[32] N. Sun, H. Liu, B. Xu, Facile synthesis of high performance hard carbon anode 
materials for sodium ion batteries, J. Mater. Chem. A 3 (2015) 20560-20566.

[33] H. G. Wang, Z. Wu, F. L. Meng, D. L. Ma, X. L. Huang, L. M. Wang, X. B. Zhang, Nitrogen-doped porous carbon nanosheets as low-cost, high-performance anode material for sodium-ion batteries, ChemSusChem 6 (2013) 56-60.

[34] Y. Cao, L. Xiao, M. L. Sushko, W. Wang, B. Schwenzer, J. Xiao, Z. Nie, L.V. Saraf, Z. Yang, J. Liu, Sodium ion insertion in hollow carbon nanowires for battery applications, Nano Lett. 12 (2012) 3783-3787.

[35] X. Zhou, Y. Guo, Highly disordered carbon as a superior anode material for room-temperature sodium-ion batteries, ChemElectroChem. 1 (2014) 83-86.

[36] X. Zhou, X. Zhu, X. Liu, Y. Xu, Y. Liu, Z. Dai, J. Bao, Ultralong cycle life sodium-ion battery anodes using a graphene-templated carbon hybrid, J Phys. Chem. C 118 (2014) 22426-22431.

[37] T. Chen, Y. Liu, L. Pan, T. Lu, Y. Yao, Z. Sun, D. H. C. Chua, Q. Chen, Electrospun carbon nanofibers as anode materials for sodium ion batteries with excellent cycle performance, J. Mater. Chem. A 2 (2014) 4117-4121.

[38] K. Tang, L. Fu, R. J. White, L. Yu, M. M. Titirici, M. Antonietti, J. Maier, Hollow carbon nanospheres with superior rate capability for sodium-based batteries, Adv. Energy Mater. 2 (2012) 873-877. 
Table 1 Microstructure parameters and composition of the carbons.

\begin{tabular}{|c|c|c|c|c|c|c|c|c|c|c|}
\hline \multirow{2}{*}{ Samples } & \multirow{2}{*}{$\begin{array}{c}\mathrm{d}_{002} \\
(\mathrm{~nm})\end{array}$} & \multirow{2}{*}{$\begin{array}{c}\mathrm{S}_{\mathrm{BET}} \\
\left(\mathrm{m}^{2} \mathrm{~g}^{-1}\right)\end{array}$} & \multirow{2}{*}{$\begin{array}{c}\mathrm{V} \\
\left(\mathrm{m}^{3} \mathrm{~g}^{-1}\right)\end{array}$} & \multirow{2}{*}{$\begin{array}{c}\mathrm{C} \\
\text { (at \%) }\end{array}$} & \multirow{2}{*}{$\begin{array}{c}\mathrm{O} \\
\text { (at \%) }\end{array}$} & \multicolumn{5}{|c|}{$\mathrm{N}($ atom \%) } \\
\hline & & & & & & Total & N-6 & N-5 & N-Q & N-oxide \\
\hline $\mathrm{NC}$ & 0.379 & 220 & 0.20 & 84.31 & 7.37 & 8.32 & 2.57 & 2.08 & 2.56 & 1.10 \\
\hline NCG700 & 0.360 & 94.1 & 0.12 & 86.56 & 5.91 & 7.54 & 2.43 & 2.28 & 2.31 & 0.43 \\
\hline NCG800 & 0.358 & 32.8 & 0.10 & 88.32 & 4.87 & 6.81 & 2.10 & 1.18 & 2.79 & 0.74 \\
\hline NCG900 & 0.351 & 35.1 & 0.12 & 89.96 & 4.45 & 5.68 & 1.82 & 0.23 & 2.92 & 0.71 \\
\hline
\end{tabular}

